\title{
Función adrenal y metabolismo lipídico en niños asmáticos tratados con budesonida
}

\author{
Mariela Paoli-de Valeri, M.C., ${ }^{(1)}$ Yajaira Zerpa-de Miliani, M.C., ${ }^{(1)}$ \\ Ezio G.Valeri-Dávila, M.C. ${ }^{(2)}$ G abriela Bellabarba, M.C ., M. en C. ${ }^{(3)}$
}

\section{Paoli-de Valeri M, Zerpa-de Miliani Y, Valeri-Dávila EG, Bellabarba G. Función adrenal y metabolismo lipídico en niños asmáticos tratados con budesonida. Salud Publica Mex 1999;41:119-123.}

\section{Resumen}

Objetivo. Evaluar el efecto de bajas dosis de budesonida inhalado sobre la función adrenal y el metabolismo lipídico en niños asmáticos. Material y métodos. Se estudiaron: 10 niños asmáticos (edad promedio, 8.6 años) tratados con budesonida inhalado (200-300 $\mu \mathrm{g} / \mathrm{día}$ ) por un lapso mayor a tres meses (grupo A); 15 niños asmáticos (edad promedio, 7.8 años) sin tratamiento esteroideo (grupo B), y 10 niños no asmáticos (grupo C). Se determinaron los niveles de cortisol basal y postestímulo con ACTH, andrógenos adrenales, lípidos y cortisol urinario. Resultados Entre los grupos A y $B$ no hubo diferencias significativas en las variables estudiadas. En los niños asmáticos (grupo A-B) el cortisol urinario fue significativamente mayor en relación con el grupo C. Los niveles de triglicéridos, colesterol total, colesterol de la lipoproteína de baja densidad e índices aterogénicos fueron mayores en el grupo de niños asmáticos, con y sin budesonida, comparados con el grupo C. Conclusiones. El tratamiento con dosis bajas de budesonida inhalado en niños asmáticos no modificó la función del eje adrenal ni el metabolismo lipídico. Los pacientes asmáticos presentaron un perfil lipídico aterogénico que podría incrementar el riesgo de enfermedad cardiovascular.

Palabras clave: asma; niño; esteroides; lípidos/metabolismo; función adrenal; Venezuela
Paoli-de Valeri M, Zerpa-de Miliani Y, Valeri-Dávila EG, Bellabarba G.

Adrenal function and lipid metabolism

in asthmatic children with budesonide treatment.

Salud Publica Mex 1999;41:119-123.

\begin{abstract}
A bstract
Objective. To assess the effect of low doses of inhaled budesonide on the adrenal function and lipid metabolism of asthmatic children. Material and methods. The study included 10 asthmatic children (mean age, 8.8 years) treated with inhaled budesonide (200-300 $\mu \mathrm{g} /$ day) for a period longer than 3 months (group A); 15 asthmatic children (mean age, 7.8 years) without steroid treatment (group B) and 10 non-asthmatic children (group C). Basal cortisol levels, as well as postACTH, adrenal androgens, lipids and urinary cortisol were determined. Results N o significant differences were detected between groups A and B in the studied variables. In asthmatic children, urinary cortisol was significantly higher than in non-asthmatic children. Triglycerides, total cholesterol, low density lipoprotein cholesterol and atherogenic index levels were higher in asthmatic children with and without budesonide treatment, compared with nonasthmatic children. Conclusions Treatment of asthmatic children with low doses of inhaled budesonide did not modify the adrenal axis function nor lipid metabolism. Asthmatic patients showed an atherogenic lipid profile which could increase the risk of cardiovascular disease.
\end{abstract}

Key words: asthma; child; steroids; lipids/metabolism; adrenal function; Venezuela

(1) Unidad de Endocrinología, Universidad de Los Andes (ULA), Instituto Autónomo Hospital Universitario de Los Andes (IAHULA), Mérida,Venezuela.

(2) Unidad de Pediatría, ULA, IAHULA, Mérida, Venezuela.

(3) Unidad de Fisiopatología, ULA, IAHULA, Mérida,Venezuela.

Fecha de recibido: 30 de junio de 1998 - Fecha de aprobado: 19 de octubre de 1998 Solicitud de sobretiros: Dra. Mariela Paoli de Valeri. Urb. La Mara, Av. 3 Caribay 122, Q ta. Mariela. Mérida,Venezuela. 
L os glucocorticoides ejercen un excelente control sobre los síntomas del asma bronquial. ${ }^{1,2}$ Los efectos adversos como la supresión del eje hipotálamo-hipófisis adrenal y las alteraciones lipídicas, entre otros, constituyen la mayor preocupación cuando se requiere del uso crónico de estos medicamentos. ${ }^{3-5} \mathrm{El}$ advenimiento de los esteroides en aerosoles tópicos como el budesonida ha transformado su uso en el tratamiento del asma, ${ }^{6}$ aunque estos aerosoles también parecen estar asociados con algunos efectos colaterales. ${ }^{7,8}$

Algunos autores han observado que los esteroides inhalados suprimen la función adrenal con dosis tanto altas $\left(800 \mu \mathrm{g} /\right.$ día) como bajas $(400 \mu \mathrm{g} / \text { día) })^{9,10} \mathrm{Sin}$ embargo, otros autores no encuentran pruebas de que los glucocorticoides inhalados reduzcan la respuesta de cortisol plasmático ante el estrés provocado por las crisis de asma. ${ }^{2}$ Igualmente, se han descrito efectos deletéreos sobre el metabolismo lipídico. Se ha informado sobre un aumento en los niveles plasmáticos de colesterol total con el uso de los esteroides inhalados, ${ }_{1,12}^{11}$ lo cual podría ser un factor de riesgo de enfermedad cardiovascular en niños que reciben altas dosis de estos medicamentos por tiempo prolongado. Por lo antes expuesto, se planteó investigar si los niños asmáticos que reciben budesonida presentan alguna alteración en la función adrenal y en el metabolismo lipídico.

\section{Material y métodos}

Entre junio de 1996 y febrero de 1997 se estudiaron 35 niños. De la consulta de neumonología del Hospital Universitario de Los Andes se seleccionaron 25 niños asmáticos, de ambos sexos, sin tratamiento esteroideo sistémico en los seis meses previos a la investigación. Los 10 niños restantes fueron no asmáticos, seleccionados de entre la población general. Se excluyeron los niños con patologías o uso de medicamentos que alteran el eje hipotálamo-hipófisis-adrenal o el metabolismo lipídico. Asimismo, se excluyeron los niños con asma no controlada a pesar del tratamiento. Los 35 niños se dividieron en tres grupos:

- Grupo A: diez niños con asma leve o moderada persistente (determinada por parámetros clíni$\cos ^{13} \mathrm{y}$ espirométricos $\left.{ }^{14}\right)$, es decir, dos varones $\mathrm{y}$ ocho mujeres, con promedio de edad de $8.6 \pm 1.2$ años (rango de 6.6-9.9 años) tratados con budesonida (Pulmicort ${ }^{\circledR}$ ), a una dosis de 200 a 300 $\mu \mathrm{g} /$ día. Para el momento del estudio todos los niños tenían más de tres meses recibiendo el tratamiento. La duración promedio del mismo fue de 10.4 meses, con un rango de 3 a 24 meses. Cin- co de los niños recibían, además, broncodilatadores: salbutamol, fenoterol y teofilina.

- Grupo B: quince niños con asma intermitente o leve persistente, esto es, siete mujeres y ocho varones, con promedio de edad de $7.8 \pm 1.7$ años (rango de 5.1-10.6 años), sin tratamiento esteroideo. Eran tratados con broncodilatadores por razón necesaria; tres de ellos recibían sulfato de salbutamol, y los 12 restantes, mezclas de teofilina, clenbuterol, fenoterol y salbutamol a dosis calculadas de acuerdo con el peso. Nunca fueron tratados con esteroides inhalados.

- Grupo C: diez niños -cuatro mujeres y seis varones- con una edad promedio de $7.3 \pm 1.2$ años (rango de 5.3-8.8 años), y no asmáticos.

A los niños seleccionados según los criterios antes mencionados y con el previo consentimiento de sus padres para la participación en la investigación, se les realizó un examen físico que incluyó toma de peso, talla y cálculo del índice de masa corporal (IMC). Se instruyó a los padres sobre la recolección de orina de 24 horas para la medición de cortisol libre; se descartó la primera orina de la mañana, de tal modo que se comenzó la recolección con la segunda orina del día, y se incluyó hasta la primera orina del día siguiente. A todas las muestras se les realizó corrección mediante la fórmula del aclaramiento de creatinina en 24 horas para corroborar una adecuada recolección de las mismas. ${ }^{15}$

Se les tomó muestra de sangre en ayuno (8:00 horas) de la vena antecubital, y el plasma y el suero se almacenaron a $-40^{\circ} \mathrm{C}$ y $-70{ }^{\circ} \mathrm{C}$, respectivamente, hasta el momento de ser analizados. Sólo a los niños asmáticos (grupos A y B) se les realizó la prueba de estimulación con hormona adrenocorticotrófica (ACTH) (Cortrosyn ${ }^{\circledR}$ ) utilizando una inyección endovenosa de $0.25 \mathrm{mg}$. Se tomó una muestra de sangre después de 60 minutos para el análisis del cortisol sérico.

En el suero se realizaron las determinaciones hormonales de cortisol, androstenediona y dehidroepiandrosterona-sulfato (DHEA-S), las cuales se efectuaron por radioinmunoanálisis (RIA), utilizando dispositivos comerciales (Diagnostic Products Corporation, Los Angeles, California). En el plasma se midieron los lípidos y las lipoproteínas: triglicéridos (TG) y colesterol total (CT), por métodos enzimáticos, y colesterol de la lipoproteína de alta densidad (C-HDL), por el método de precipitación con solución de cloruro de manganeso. ${ }^{16}$ El colesterol de la lipoproteína de baja densidad (C-LDL) se estimó por la fórmula de Friedewald ${ }^{17}$ y se realizaron los cálculos de los índices aterogénicos CT/C-HDL y C-LDL/C-HDL. 


\section{A nálisis estadístico}

Los resultados de las variables se presentan en promedios \pm desviación estándar $(\overline{\mathrm{X}} \pm \mathrm{DE})$. La determinación de las diferencias entre los grupos se realizó mediante la aplicación del ANOVA y las pruebas de Fisher y Scheffé, o bien, de la prueba $t$ de Student de dos colas para datos no pareados según el caso. Se tomó como significativo un valor de $p<0.05$. Los cálculos se realizaron con la ayuda del programa de estadística STAT VIEW $512 \mathrm{TM}+$.

\section{Resultados}

En el cuadro I se muestran los datos clínicos de los niños de cada grupo de estudio. La edad, el peso, la talla y el IMC no varían entre los grupos.

En el cuadro II se presenta el perfil de la función adrenal y del metabolismo lipídico obtenido en niños asmáticos tratados con budesonida (grupo A) y sin budesonida (grupo B). Los niveles séricos de cortisol basal, cortisol postestímulo con ACTH, androstenediona, DHEA-S y el cortisol en orina de 24 horas no son diferentes entre los grupos A y B. De igual forma, los niveles plasmáticos de TG, CT, C-LDL, C-HDL y los índices aterogénicos CT/C-HDL y C-LDL/C-HDL no muestran diferencias significativas entre los niños asmáticos tratados o no con budesonida.

No habiéndose obtenido ninguna diferencia entre los niños asmáticos tratados y no tratados con budesonida, se realizó la comparación entre el grupo de todos los niños asmáticos (grupo A-B) y los niños controles (grupo C). Como se puede observar en el cuadro III, el perfil de la función adrenal en los niños asmáticos fue similar al de los controles, a excepción de un

Cuadro I

Datos clínicos de los niños asmáticos tratados CON Y SIN BUDESONIDA Y DE LOS NIÑOS CONTROL. Hospital Universitario de Los Andes, Mérida, Venezuela, 1996-1997

\begin{tabular}{lrcr} 
& $\begin{array}{r}\text { Grupo } A \\
\text { No.=10 } \\
(\bar{X} \pm D E)\end{array}$ & $\begin{array}{r}\text { Grupo B } \\
\text { No. }=15 \\
(\bar{X} \pm D E)\end{array}$ & $\begin{array}{r}\text { Grupo } C \\
\text { No. }=10 \\
(\bar{X} \pm D E)\end{array}$ \\
Edad (años) & $8.6 \pm 1.2$ & $7.8 \pm 1.7$ & $7.3 \pm 1.2$ \\
\hline Peso $(\mathrm{kg})$ & $27.0 \pm 7.8$ & $25.6 \pm 7.3$ & $24.6 \pm 3.9$ \\
\hline Talla $(\mathrm{cm})$ & $130.0 \pm 8.5$ & $125.2 \pm 12.6$ & $124.1 \pm 8.0$ \\
\hline IMC $\left(\mathrm{kg} / \mathrm{m}^{2}\right)$ & $15.9 \pm 2.8$ & $16.0 \pm 1.8$ & $15.9 \pm 0.8$
\end{tabular}

Grupo A: niños asmáticos tratados con budesonida Grupo B: niños asmáticos tratados sin budesonida Grupo $C$ : niños control $D E:$ desviación estándar
Cuadro II

Perfil de función adRenal y metabolismo lipídico EN NIÑOS ASMÁTICOS TRATADOS CON Y SIN BUDESONIDA. Hospital Universitario de Los Andes, Mérida, Venezuela, 1996-1997

\begin{tabular}{lcc} 
& $\begin{array}{r}\text { Grupo A } \\
\text { No.=10 } \\
(\bar{X} \pm D E)\end{array}$ & $\begin{array}{c}\text { Grupo B } \\
\text { No. }=15 \\
(\bar{X} \pm D E)\end{array}$ \\
Cortisol basal $(\mu \mathrm{g} / \mathrm{dl})$ & $10.3 \pm 5.2$ & $10.5 \pm 4.5$ \\
\hline Cortisol postestímulo $(\mu \mathrm{g} / \mathrm{dl})$ & $34.1 \pm 8.4$ & $37.0 \pm 9.8$ \\
\hline Cortisol orina $(\mu \mathrm{g} / 24 \mathrm{hs})$ & $22.5 \pm 12.5$ & $19.2 \pm 10.6$ \\
\hline Androstenediona $(\mathrm{ng} / \mathrm{ml})$ & $0.47 \pm 0.38$ & $0.34 \pm 0.30$ \\
\hline DHEA-S $(\mu \mathrm{g} / \mathrm{dl})$ & $56.7 \pm 26.5$ & $51.2 \pm 27.6$ \\
\hline $\mathrm{TG}(\mathrm{mg} / \mathrm{dl})$ & $126.5 \pm 51.0$ & $111.4 \pm 38.9$ \\
\hline $\mathrm{CT}(\mathrm{mg} / \mathrm{dl})$ & $158.2 \pm 20.8$ & $173.1 \pm 33.3$ \\
\hline $\mathrm{C}-\mathrm{LDL}(\mathrm{mg} / \mathrm{dl})$ & $93.9 \pm 25.8$ & $112.4 \pm 33.4$ \\
\hline $\mathrm{C}-\mathrm{HDL}(\mathrm{mg} / \mathrm{dl})$ & $39.1 \pm 11.4$ & $38.3 \pm 6.0$ \\
\hline $\mathrm{CT} / \mathrm{C}-\mathrm{HDL}$ & $4.33 \pm 1.22$ & $4.58 \pm 0.86$ \\
\hline $\mathrm{C}-\mathrm{LDL} / \mathrm{C}-\mathrm{HDL}$ & $2.60 \pm 0.94$ & $2.96 \pm 0.82$
\end{tabular}

Grupo A: niños asmáticos tratados con budesonida Grupo B: niños asmáticos tratados sin budesonida DE: desviación estándar

\section{Cuadro III}

Perfil de función adRenal y metabolismo lipídico EN TODOS LOS NIÑOS ASMÁTICOS Y EN LOS NIÑOS control. Hospital Universitario de Los Andes, Mérida, Venezuela, 1996-1997

$\begin{array}{ll}\text { Grupo A-B } & \text { Grupo C } \\ N 0 .=25 & \mathrm{~N} 0 .=10 \\ (\bar{X} \pm D E) & (\bar{X} \pm D E)\end{array}$

\begin{tabular}{lcc} 
Cortisol basal $(\mu \mathrm{g} / \mathrm{dl})$ & $10.4 \pm 4.7$ & $15.0 \pm 9.4$ \\
\hline Cortisol orina $(\mu \mathrm{g} / 24 \mathrm{hs})$ & $20.5^{*} \pm 3.0$ & $12.2 \pm 3.0$ \\
\hline Androstenediona $(\mathrm{ng} / \mathrm{ml})$ & $0.39 \pm 0.33$ & $0.29 \pm 0.28$ \\
\hline DHEA-S $(\mu \mathrm{g} / \mathrm{dl})$ & $53.4 \pm 26.7$ & $65.8 \pm 69.0$ \\
\hline $\mathrm{TG}(\mathrm{mg} / \mathrm{dl})$ & $117.4^{*} \pm 43.8$ & $82.1 \pm 20.6$ \\
\hline $\mathrm{CT}(\mathrm{mg} / \mathrm{dl})$ & $167.2^{\ddagger} \pm 29.4$ & $132.1 \pm 18.9$ \\
\hline $\mathrm{C}-\mathrm{LDL}(\mathrm{mg} / \mathrm{dl})$ & $105.0^{\S} \pm 31.4$ & $78.1 \pm 16.4$ \\
\hline $\mathrm{C}-\mathrm{HDL}(\mathrm{mg} / \mathrm{dl})$ & $38.6 \pm 8.3$ & $40.7 \pm 8.3$ \\
\hline $\mathrm{CT} / \mathrm{C}-\mathrm{HDL}$ & $4.48^{\ddagger} \pm 1.00$ & $3.32 \pm 0.56$ \\
\hline $\mathrm{C}-\mathrm{LD} \mathrm{L} / \mathrm{C}-\mathrm{HDL}$ & $2.82^{\S} \pm 0.87$ & $1.99 \pm 0.59$
\end{tabular}

Grupo A-B: todos los niños asmáticos

Grupo $C$ : niños control

$D E:$ desviación estándar

$* p<0.05$

$\ddagger p=0.001$

$\S p=0.01$

aumento significativo del cortisol en orina de 24 horas $(p<0.05)$. En relación con el metabolismo lipídico se aprecia que, con excepción del C-HDL, todas las variables del lipidograma están significativamente aumen- 
tadas. Individualmente, 13 pacientes (52\%) tuvieron niveles superiores al máximo obtenido en los niños del grupo control $(108 \mathrm{mg} / \mathrm{dl})$, y seis pacientes, esto es, tres que recibían budesonida y tres que no la recibían (24\%), presentaron niveles mayores de $150 \mathrm{mg} / \mathrm{dl}$, que es el límite superior aceptado para estas edades según el informe del panel de expertos en el tratamiento de las hipertrigliceridemias. ${ }^{18}$ En ocho de los niños asmáticos (32\%) se presentaron niveles de CT superiores al valor máximo de los niños control (168 mg/dl), y tres pacientes (12\%, todos del grupo sin budesonida) presentaron niveles de CT mayores de $200 \mathrm{mg} / \mathrm{dl}$, que es el límite máximo para esta edad según el panel de expertos. ${ }^{19}$ Mayor alteración que en el CT, se observó en los niveles de C-LDL, donde 11 de los niños asmáticos $(44 \%)$ tuvieron niveles superiores al valor máximo de los controles ( $105 \mathrm{mg} / \mathrm{dl}$ ), y cuatro pacientes, uno con budesonida y tres sin el esteroide $(16 \%)$, presentaron niveles de C-LDL mayores de $130 \mathrm{mg} / \mathrm{dl}$, que es el límite superior normal para los niños. ${ }^{19}$

\section{Discusión}

En este trabajo se observó que en los niños asmáticos tratados con budesonida inhalado a dosis menores o iguales de $300 \mu \mathrm{g} /$ día, no se produjeron alteraciones en la función adrenal al compararlos con un grupo de niños asmáticos, similar en edad, peso y talla, pero sin el esteroide. Estos hallazgos corroboran los resultados de otros autores, ${ }^{2,5,8,20}$ quienes indicaron la eficacia y la seguridad de los esteroides inhalados, sin efecto sobre la función adrenal, siempre y cuando sean utilizados en dosis apropiadas, generalmente suficientes para controlar los síntomas del asma bronquial. Dosis mayores de $400 \mu \mathrm{g} /$ día de budesonida se han asociado con supresión de la función adrenal. ${ }^{9}$

Llama la atención que en todos los niños asmáticos (grupo A-B), la excreción de cortisol urinario fue significativamente más alta que en los niños no asmáticos (grupo C). Los informes disponibles establecen la comparación entre niños asmáticos con y sin tratamiento esteroideo sin hacer referencia a niños sanos. ${ }^{5,10,21}$ Los resultados de este estudio sugieren que el asma bronquial per se, o los medicamentos asociados como son los broncodilatadores, podrían favorecer la excreción urinaria de cortisol.

Respecto al perfil lipídico, los resultados no mostraron modificaciones en el metabolismo lipídico atribuibles a los esteroides inhalados. Se ha informado de aumentos significativos en los niveles de colesterol total, ${ }_{11}^{11}$ pero en los casos en los que se han utilizado dosis más elevadas del medicamento ( $>800 \mu \mathrm{g} /$ día). Es inte- resante destacar que los niños asmáticos (con y sin budesonida) presentaron un perfil lipídico diferente al de los no asmáticos, caracterizado por valores significativamente más elevados de TG, CT, C-LDL y de los índices aterogénicos. En el análisis individual, un grupo importante de los niños asmáticos presentaron valores de lípidos plasmáticos por encima de los límites superiores establecidos, ${ }^{18,19}$ en particular $24 \%$ de esos niños presentaron niveles de TG por arriba de $150 \mathrm{mg} / \mathrm{dl}, 12 \%$ mostraron niveles mayores de 200 $\mathrm{mg} / \mathrm{dl}$ de CT y 16\% de los niños asmáticos tuvieron niveles de C-LDL mayores de $130 \mathrm{mg} / \mathrm{dl}$. Es evidente que las diferencias observadas en los lípidos no son atribuibles al uso de los esteroides inhalados; sin embargo, podrían ser secundarias al efecto de los broncodilatadores por su acción catecolaminérgica, ${ }^{22} \mathrm{o}$ bien, a la enfermedad misma. Las catecolaminas pueden aumentar los niveles de colesterol plasmático, al estimular la actividad de la 3hidroxi-3metilglutaril CoA, enzima que controla la tasa de síntesis de colesterol. Asimismo, incrementan los valores de TG pues favorecen la movilización de ácidos grasos libres a partir del tejido adiposo, permitiendo mayor disponibilidad de los mismos como sustrato para la síntesis de TG a nivel hepático. ${ }^{23}$ En ese sentido, existen pocos informes publicados, aunque Lehtonen y colaboradores $^{24}$ demostraron que el salbutamol (agonista B2adrenérgico) produjo una ligera elevación del CT y del C-LDL, lo cual apoyaría los presentes resultados. Por otra parte, se han notificado niveles más bien disminuidos de CT, C-LDL, TG, Apo B y niveles elevados de C-HDL y Apo AII en adultos con asma bronquial sin tratamiento específico, ${ }^{25}$ de allí que los cambios observados en el perfil lipídico de los pacientes de este estudio probablemente no sean consecuencia de la enfermedad misma, sino de la utilización de broncodilatadores para el tratamiento del asma bronquial. Son necesarios más estudios en este sentido para poder determinar definitivamente la posible asociación entre el uso de broncodilatadores B2-adrenérgicos y las alteraciones lipídicas.

Se concluye que la administración de budesonida inhalado, a dosis de 200 a $300 \mu \mathrm{g}$ / día, en niños asmáticos, no modifica la función del eje adrenal ni el metabolismo lipídico; por lo tanto, estos medicamentos son una buena alternativa terapéutica en el manejo crónico del asma bronquial sin riesgo de complicaciones en las esferas mencionadas. Los pacientes asmáticos presentaron alteración en el perfil lipídico, con tendencia hacia un patrón aterogénico, por lo que, conociendo la relación que existe entre la dislipidemia y la arterioesclerosis, ${ }^{26}$ se recomienda realizar a todo niño asmáti- 
co un lipidograma y, en caso de hallar alteración, tomar las medidas higiénico-dietéticas necesarias para evitar que en el largo plazo se desarrolle una enfermedad cardiovascular.

\section{Referencias}

1. Chung K, Clark T: Corticosteroids. En: Clark T, Godfrey S, Lee T, eds. Asthma. Londres: Chapman and Hall Medical, 1992:416-448.

2. Barnes P. Inhaled glucocorticoids for asthma. N Engl J Med 1995;332: 868-875.

3. Szefler S. Glucocorticoid therapy for asthma: Clinical pharmacology. J Allergy C lin Immunol 1991;88:147-165.

4. Frew A, Holgate $S$. Clinical pharmacology of asthma. Implication for treatment. D rugs 1993;46:847-862.

5. Priftis $K$, Milner $D$, Conway $E$, Honour J. Adrenal function in asthma. Arch D is Child 1990;65:838-840.

6. Toogood J, Baskerville J, Jennings B. Bioequivalent doses of budesonida and prednisona in moderate and severe asthma. J Allergy Clin Immunol 1989;84:688-700.

7.W olthers 0 , Pedersen $\mathrm{S}$. C ontrolled study of linear growth in asthmatic children during treatment with inhaled glucocorticosteroids. Pediatrics 1992:89:839-842.

8. D olan L, Kesarwala H, H olroyde J, Fisher T. Short-term high-doses, systemic steroids in children with asthma: The effect on the hypothalamicpituitary-adrenal axis. J Allergy C lin Immunol 1987;80:81-87.

9.Volovitz B,Amir J, Malik H, Kauschansky A, Varsano I. Growth and pituitary adrenal function in children with severe asthma treated with inhaled budesonida. N Engl J Med 1993;329:1703-1708.

10. Z waan C, $O$ dink R, D elmare-Vander W aal H.A cute adrenal insuffiency after discontinuation of inhaled corticosteroids therapy. Lancet 1992; 340:1289-1290.

11. Turpeinem M, Sorva R, Juntunen-Backman K. C hanges in carbohidrate and lipid metabolism in children with asthma inhaling budesonida. J Allergy Clin Immunol 1991;88:384-389.

12. H anania N, Chapman K, Kesten S.A dverse effects of inhaled corticosteroids. Am J Med 1995;98:196-206.

13. Joint task force of the A cademy and the American College of Alergy and Immunology. Classification of asthma severity. J Alergy Clin Immunol 1995; 96:749-751.
14. Manual de espirometría Fukuda-Sanyo SP 200. Escala de obstrucción bronquial. Tokio, 1986.

15. Gordillo G, Mota F, Velázquez J. Pruebas de función renal y metodología de laboratorio. En: Gordillo G, M ota F,Velázquez. N efrología pediátrica. México, D.F.: Ediciones Médicas del Hospital Infantil de México, 1980: 485-503.

16. IshikawaT, Brazier J, Steiner P, Steward L, G artside B, G lueck C .A study of the heparin-manganese chloride methods for determination of plasma alpha-lipoprotein cholesterol concentration. Lipids 1976;11:628-639.

17. Friedewald W, Levy R, Frederickson D. Estimation of the concentration of low density lipoprotein cholesterol in plasma, without use of the preparate ultracentrifuge. Clin Chem 1972;18:499-515.

18. $\mathrm{N}$ ational Heart, Lung and Blood Institute Consensus Development Panel:Treatment of hypertriglyceridemia. JA MA 1984;251:1196-1200.

19. Expert Panel on Blood Cholesterol Levels in Children and Adolescents. Report of the Expert Panel on Blood Cholesterol Levels in Children and Adolescents. U.S. Department of Health and Human Services, Public Health Service, N ational Institutes of H ealth (Publication 91-2732), 1991.

20. Goldstein D, Konig P. Effect of inhaled beclomethasone dipropionate on hypothalamic-pituitary-adrenal axis function in children with asthma. Pediatrics 1983;72:60-64.

21. Tabachinik E, Zadik Z. D iurnal cortisol secretion during therapy with inhaled beclomethasone dipropionate in children with asthma. J Pediatr 1991;118:294-297.

22. Hoffman B, Lefkowitz R. C athecholamines, simpathomimetic drugs, and adrenergic receptor antagonists. En: Hardman J, Limbird L, Molinoff $P$, Ruddon R, Goodman A, eds. Goodman \& Gilman's the pharmacological basis of therapeutics. 9a. edición. Nueva York: Mc Graw Hill, 1996:199248.

23. Landsberg L,YoungJ.C atecholamines and the adrenal medulla. En:W ilson J, Foster D, eds.W illiams textbook of endocrinology. 8a. edición. Filadelfia: W.B. Saunders Company, 1992:621-705.

24. Lehtonen A, ViikasiY, Sallinen V, Elo Y. Effect of beta2-adrenergic stimulation on serum lipids. Int J C lin Pharmacol Ther Toxicol 1982;20:530-531. 25. N akasawa K, Yamazaki N, Taniguachi M. Changes of serum lipids and high density lipoprotein levels in patients with bronchial asthma. N ippon Kyobu Shikkan Gakkai Zasshi 1991;29:435-438.

26. Rose G, Shipley M. Plasma cholesterol concentration and death from coronary heart disease: 10 years results of the W hitehall Study. Br Med J 1986;293:306-307. 